\title{
Treatment of pathological tooth migration using Non- surgical periodontal therapy
}

\begin{abstract}
:
Migration or drifting of teeth is always multifactorial. This is one of the major concerns in the field of dentistry. On long standing periodontal disease this pathological migratory phenomenon occurs. Treating this pathological tooth migration requires some of the modalities like periodontal therapy/ orthodontic therapy/ restorative therapy. But in some cases periodontal therapy alone plays an important role in spontaneous correction of PTM. Present case report also demonstrate the spontaneous correction of mandibular anterior tooth proclination and slight amount of open bite with non- surgical periodontal therapy (periodontal therapy) alone
\end{abstract}

Key words: Chronic Periodontitis, Non-Surgical periodontal therapy, Tooth Migration, Tooth Drifting, Spontaneous correction

\section{Introduction:}

Pathological Tooth Migration (PTM) is one of the manifestations of long standing periodontal disease. Whenever there is an alteration of balance among factors due to periodontal disease, tooth displacement results this is called pathological tooth migration.[1] Generally some factors like plaque and calculus, pressure due to inflamed tissue in pockets, underlying bone loss, abnormal brushing habits, mouth breathing, nail biting, pressure from musculature of oral cavity, aberrant frenal attachments, pipe smoking, gingival enlargement, usage of some instruments, iatrogenic factors cause tooth movement.[2] It has a prevalence of about $30 \%$ to $55 \% .3 \mathrm{~A}$ tooth can be diagnosed with pathological tooth migration only when there was flaring of tooth, extrusion, protrusion, rotation, drifting into edentulous spaces, or combination of conditions.

Even though there is awareness regarding the pathological migration, scientific evidence of treating the problem is very limited so the present case report describes the impact of nonsurgical periodontal therapy in correcting (spontaneous repositioning of tooth) the PTM without adjunctive use of orthodontic therapy and restorative treatments.[4]

\begin{tabular}{|l|l|}
\hline \multicolumn{3}{|c|}{ Access this article online } \\
\hline \multirow{2}{*}{$\begin{array}{l}\text { Website: } \\
\text { www.ujds.in }\end{array}$} & Quick Response Code \\
\hline $\begin{array}{l}\text { DOI: } \\
\text { https://doi.org/10.21276/10.21276/ujds.2020.6.2.13 }\end{array}$ & \\
\hline
\end{tabular}

PTM is one of the major concerns regarding aesthetics, as it occurs in the anterior region. There will be migration or drifting of teeth that cause disturbance in social balance and cannot move in society so it should be attempted to correct. Improvement in oral condition motivates the patient regarding the appearance and aesthetic problem solving.

\section{Case History:}

An 18 year systemically healthy, non-smoker female patient came to the Department of Periodontics, Institute of Dental Sciences, Bareilly with a chief complaint of bleeding from gums and bad breath since 6 months. On clinical examination, there was moderate amount of plaque and sub-gingival calculus present. Spontaneous bleeding with exudate was seen during probing. (Figure: 1) There was generalized gingival inflammation, with proclination of lower 41 and slight amount of anterior open bite. Periodontal pockets of $4 \mathrm{~mm}$ to $5 \mathrm{~mm}$ were also seen while examining with periodontal probe. Patient also has congenital absence maxillary lateral incisors.

\section{${ }^{1}$ AGARWAL A, ${ }^{2}$ GUMMALURI S S \\ Institute of Dental Sciences, Bareilly}

Address for Correspondence: Dr. Ashish Agarwal Reader, Institute of Dental Sciences, Bareilly

Email id: drashish.aag@gmail.com

Received : 04 March 2020, Published : 31 August 2020

How to cite this article: Agarwal A, Gummaluri S S (2020). Treatment of Pathological Tooth Migration using Non- Surgical Periodontal Therapy. UNIVERSITY JOURNAL OF DENTAL SCIENCES, 6(2): 112-14. 
Full mouth scaling and root planing was performed on the same day of examination with both ultrasonic scalers and hand instrumentation. Oral hygiene instructions were given and $0.12 \%$ chlorhexidine solution was prescribed twice daily for a period of two weeks. Antibiotic prophylaxis was also prescribed (Amoxicillin 500mg thrice daily for 5 days and Diclofenac sodium+ paracetamol twice daily for 5days) Patient was recalled after two weeks (Figure: 2) for reevaluation. During this phase gingival inflammation, bleeding from gums, pockets were subsided. Then the patient was kept under follow-up for a period of 3 months (Figure: 3 ). During this period, there was spontaneous correction of proclination of 41 with slight correction of anterior open bite. Thus there was an improvement in clinical condition of the patient.

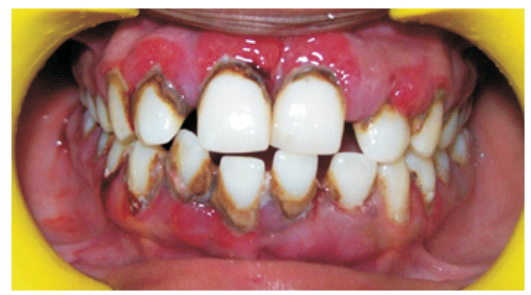

Figure: 1- Initial Pre- Operative

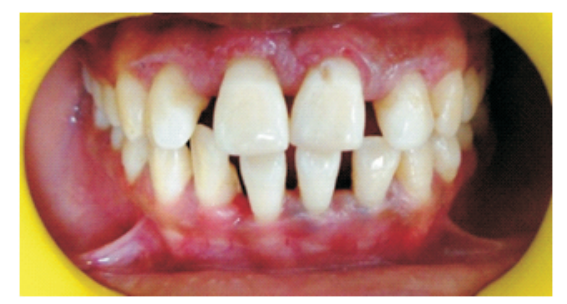

Figure: 2- Two weeks Post- Operative after Non- Surgical Periodontal therapy

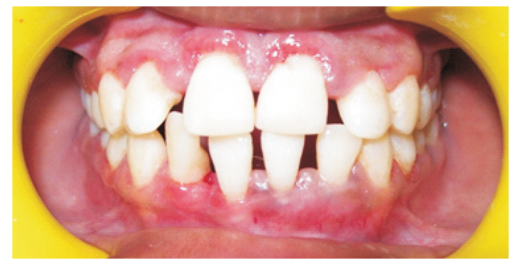

Figure: 3- Three months Post- Operative image where there was correction of proclination of 41

\section{Discussion:}

There are several possible mechanisms for explaining PTM. Plaque and calculus accumulation is one of the causes for pathological migration in addition to underlying periodontal disease. Mild cases of PTM can be corrected by normal scaling and root planing. Kim et al 4 2012, Rohtagi et al 1 in their studies stated that bone loss was the primary cause; established a relation between PTM, clinical attachment level and gingival inflammation. In the present case report there was a severe gingival inflammation which was reduced after non- surgical periodontal therapy. Thus proclination of 41 was corrected on its own and improvement in closure of open bite.

In a study by Oh SL et al 5 stated that transseptal fibers play an important role in tooth migration. Some other studies like Agarwal N6, Dadlani H et al7also found that at initial cases PTM can be corrected on its own either by surgical or nonsurgical therapy. In the present case report we have tried for non-surgical therapy and achieved good result.

Treatment of Pathological tooth migration is a multiple approach like Periodontal, orthodontic and restorative. Based on severity of PTM , treatment modalities vary from simple scaling and root planing to flap surgery ( periodontal); combined periodontal and orthodontic treatments; orthodontic treatment alone; restorative treatments (either extraction and replacement or planning a fixed prosthesis after proper treatment planning).[4] In present case, proper closure of anterior spacing can be done with orthodontic treatment. Patient was not willing for fixed orthodontic treatment so we planned a non- surgical therapy and kept under a follow up therapy. In this proclination of 41 is reduced and spontaneous repositioning occurred to most extent; healing of tissue occurred at a rapid rate. This was in accordance with the study conducted by Kumar et al 8 where he reported the spontaneous repositioning of tooth without orthodontic treatment so a new terminology called reactive positioning was introduced in the periodontal literature.

\section{Conclusion:}

Thus from the present case report we can conclude that only periodontal therapy can help in correcting the malpositioning, mild spacing of teeth in the anterior region to some extent. But further clinical studies are needed for better explanation, establishment of relation regarding the Pathological migration.

\section{References:}

1. Rohatgi S, Narula SC, Sharma RK, Tewari S, Bansal P. Clinical evaluation of correction of pathologic migration with periodontal therapy. Quintessence Int 2011;42:22-30.

2. Brunsvold MA. Pathologic tooth migration. J Periodontol 2005;76:859-66

3. Byrne PJ, Irwin C, Mullali B, Allen E, Ziada H. Periodontal problems associated with compromised anterior teeth. Dent Update 2008;35:21-2. 
University J Dent Scie 2020; Vol. 6, Issue 2

4. Kim YI, Kim MJ, Choi JI, Park SB. A multidisciplinary approach for the management of pathologic tooth migration in a patient with moderately advanced periodontal disease. Int J Periodontics Restorative Dent 2012;32:225-30.

5. Oh SL. An interdisciplinary treatment to manage pathologic tooth migration: A clinical report. J Prosthet Dent 2011;106:153-8.

6. Agrawal N, Siddani PS. Reactive positioning of pathologically migrated tooth following non-surgical periodontal therapy. Indian $\mathrm{J}$ Dent Res 2011;22:591-3.

7. Dadlani H, Ramachandra SS, Mehta DS. Spontaneous correction of pathologically migrated teeth with therapy alone. J Indian SocPeriodontol 2013;17:531-4.

8. Kumar V, S A, Thomas CM. Reactive repositioning of pathologically migrated teeth following periodontal therapy. Quintessence Int 2009;40:355-8 